\title{
Solving Measurement Problems with an Answer-Until-Correct Scoring Procedure
}

\author{
Rand R. Wilcox \\ University of Southern California
}

Answer-until-correct (AUC) tests have been in use for some time. Pressey (1950) pointed to their advantages in enhancing learning, and Brown (1965) proposed a scoring procedure for AUC tests that appears to increase reliability (Gilman \& Ferry, 1972; Hanna, 1975). This paper describes a new scoring procedure for AUC tests that (1) makes it possible to determine whether guessing is at random, (2) gives a measure of how "far away" guessing is from being random, (3) corrects observed test scores for partial information, and (4) yields a measure of how well an item reveals whether an examinee knows or does not know the correct re-

\begin{abstract}
sponse. In addition, the paper derives the optimal linear estimate (under squared-error loss) of true score that is corrected for partial information, as well as another formula score under the assumption that the Dirichlet-multinomial model holds. Once certain parameters are estimated, the latter formula score makes it possible to correct for partial information using only the examinee's usual numbercorrect observed score. The importance of this formula score is discussed. Finally, various statistical techniques are described that can be used to check the assumptions underlying the proposed scoring procedure.
\end{abstract}

When an examinee responds to a multiple-choice test item, the response made might not reflect his/her true state. The most obvious example, and the one of central concern here, is that an examinee might guess the correct response without knowing what it really is. The common solution to this problem is to assume that guessing is at random. That is, if there are $t$ alternatives from which to choose, and only one is correct, the probability of a correct response when the examinee does not know is $1 / t$. Simultaneously, however, it is recognized that to assume random guessing is indefensible. One possibility is that an examinee might be able to eliminate one or more distractors without knowing the correct response. In support of this possibility are empirical investigations of formula scoring in which it was found that the probability of guessing is substantially higher than would be expected when random guessing occurs (Bliss, 1980; Cross \& Frary, 1977). Although it might still be assumed that guessing is at random, this can have serious consequences in terms of test accuracy (e.g., Weitzman, 1970; Wilcox, 1980).

The purpose of this paper is to examine how an answer-until-correct (AUC) testing procedure might be used to take into account the effects of guessing. One advantage of the proposed scoring procedure is that its efficacy can be empirically checked in several different ways. The model contains

APPLIED PSYCHOLOGICAL MEASUREMENT

Vol. 5, No. 3, Summer 1981, pp. 399-414

(c) Copyright 1981 Applied Psychological Measurement Inc. 0146-6216/81/030399-16\$1.80 
number-correct scoring as well as the assumption of random guessing, as a special case. Thus, as will be illustrated, when observed test scores suggest that the model holds, the appropriateness of the two more common scoring procedures can be checked. On a related matter, the model can be used to test whether items are "ideal" in the sense defined by Weitzman (1970); this means that a random guessing assumption can be tested. Using the entropy function, it is also possible to measure how "close" the probability of guessing is to $1 / t$. This is important because when the probability is not close to $1 / t$, this suggests it might be possible to improve the distractors which in turn will improve test accuracy. The exact sense in which this is true is explained below. Another advantage of the model is that it yields a measure of test accuracy that is not ordinarily available. Two new formula scores are also derived, the advantages and disadvantages of which are discussed below.

A scoring rule for an AUC test has been proposed by Brown (1965) and has been empirically investigated by Gilman and Ferry (1972) and Hanna (1975), who found it to be more reliable than number-correct scoring. Moreover, an AUC testing procedure has been advocated from the standpoint of enhancing learning (Pressey, 1950). The goal in this paper is to propose a different scoring rule that corrects for partial information.

\section{Assumptions}

It is assumed that when an examinee responds to an achievement test item, he/she can be described as either knowing or not knowing the correct response. In the terminology of Reulecke (1977), this means that the model includes a binary structure variable or, following Harris and Pearlman (1978), that examinees are described in terms of a dichotomized latent trait. It can also be said that an examinee either has or has not acquired the "psychological structure" of a task (Spada, 1977). This means that the model is deterministic in the sense that if an examinee's latent state is known, and if there are no errors at the item level, it would be known whether an examinee would produce a correct response. The model, however, includes what Reulecke (1977) calls an intensity variable; in particular, it is assumed that an examinee who does not know the correct answer might give a correct response. The probability of this event is unknown, but it can be estimated with the scoring formula and probability model described below.

Following Horst (1933), it is assumed that when an examinee does not know, he/she can eliminate at most $t-2$ distractors from consideration. Once these distractors are eliminated, the examinee chooses an answer at random from among those that remain. An examinee who knows always gives the correct response. Finally, an AUC scoring procedure is assumed. This means that an examinee responds to a test item until the correct alternative is chosen.

\section{Three Types of Guessing}

Three types of guessing can be described. The first, Type I guessing, applies to a situation in which randomly sampled examinees respond to the same multiple-choice item. In this case, guessing is defined as the probability of a correct response, given that the randomly sampled examinee does not know. The second, or Type II guessing, is defined in terms of a single examinee responding to an item randomly sampled from some item domain. The rate of guessing for the examinee is the probability of a correct response to a randomly sampled item that he/she does not know. The third, Type III guessing, is the probability of a correct response over independent repeated trials where a single examinee responds to a specific item he/she does not know. Wilcox (1977a) has examined some latent structure models that are relevant to this case, but there are some practical difficulties (Wilcox, 1979) that limit their use. Only Type I and Type II guessing are considered in this paper. 


\section{A Model for AUC Tests and Type I Guessing}

Consider a randomly sampled examinee responding to a specific test item using an AUC test. For convenience, particular attention is given to the case in which the multiple-choice test item has $t=4$ alternatives from which to choose, one of which is correct. The results are readily extended to any value of $t$. Based on the above assumptions, the examinee belongs to one of $t=4$ mutually exclusive groups. In particular, the examinee either knows the correct response or can elinuinate 0,1 , or 2 distractors. Let $\zeta$ be the proportion of examinees who know, and let $\zeta_{i}$ be the proportion of examinees who can eliminate $i$ distractors. The probability of a correct response the first time a randomly selected examinee chooses an alternative is

$$
P_{1}=\zeta+\zeta_{0} / 4+\zeta_{1} / 3+\zeta_{2} / 2
$$

The probability of an incorrect response on the first choice and a correct response on the second is

$$
P_{2}=\zeta_{0} / 4+\zeta_{1} / 3+\zeta_{2} / 2
$$

The probability of two misses and then a correct response is

$$
P_{3}=\zeta_{0} / 4+\zeta_{1} / 3
$$

and the probability of three incorrect responses is

$$
P_{4}=\zeta_{0} / 4
$$

More generally,

$$
P_{i}=\sum_{j=0}^{t-i} \zeta_{j} /(t-j),
$$

where $i=2, \ldots, t$.

For a random sample of $N$ examinees let $x_{i}$ be the number who correspond to the event associated with $p_{i}$. For example, $x_{1}$ is the number of examinees who are correct on the first alternative chosen, and $x_{2}$ is the number of examinees who are incorrect and then correct. The $x_{i}$ 's have a multinomial probability function given by

$$
\left[\begin{array}{l}
N \\
x
\end{array}\right] P_{1}^{x_{1}} p_{2}^{x_{2}} p_{3}^{x_{3}} p_{4}^{x_{4}}
$$

where

$$
\left(\begin{array}{l}
N \\
x
\end{array}\right)=N ! /\left(x_{1} ! x_{2} ! x_{3} ! x_{4} !\right), x_{4}=N-x_{1}-x_{2}-x_{3}, \quad \Sigma p_{i}=1 .
$$

Since $\zeta=p_{1}-p_{2}$,

$$
\hat{\zeta}=\left(x_{1}-x_{2}\right) / N
$$

is an unbiased estimate of $\zeta$. From Zehna (1966) it also follows that $\hat{\zeta}$ is an unrestricted maximum likelihood estimator. Proceeding in a similar manner also yields unbiased, unrestricted maximum likelihood estimates of the $\zeta_{i}$ 's, namely,

$$
\begin{aligned}
& \hat{\zeta}_{0}=4 x_{4} / N \\
& \hat{\zeta}_{1}=3\left(x_{3}-x_{4}\right) / N
\end{aligned}
$$




$$
\hat{\zeta}_{2}=2\left(x_{2}-x_{3}\right) / N
$$

Note that the model assumes that

$$
P_{1} \geq P_{2} \geq P_{3} \geq P_{4} \text {. }
$$

Maximum likelihood estimates of the $\zeta^{\prime}$ s are available under this restriction of the $p_{i}$ 's, as noted by Barlow, Bartholomew, Bremner, and Brunk (1972). For example, the maximum likelihood estimate of $\zeta$, assuming Equation 12 holds, is given by Equation 8 when $x_{1} \geqslant x_{2}$, and it is $\hat{\zeta}=0$ otherwise.

\section{Using the Model to Analyze Achievement Test Items}

Macready and Dayton (1977) have described a probability model based on Type I guessing that might be used to analyze mastery tests consisting of equivalent items. This section illustrates how the above model can be used to analyze achievement test items in a similar but different fashion.

Suppose, as is customary, it is decided that an examinee knows the correct response if the first alternative chosen is the correct answer and that, otherwise, the examinee does not know. In this case a test constructor would like to know the accuracy of the decision about a typical examinee based on his/her response.

The cells in Table 1 give the probability of the four possible outcomes when an examinee responds to an item. Thus, for a randomly sampled examinee, the probability of a correct decision about an examinee's latent state is the proportion of agreement in Table 1, namely,

$$
P=\zeta+3 \zeta_{0} / 4+2 \zeta_{1} / 3+\zeta_{2} / 2
$$

An unrestricted maximum likelihood estimate of $P$ is just

$$
\hat{\mathbf{p}}=\zeta+3 \hat{\zeta}_{0} / 4+2 \hat{\zeta}_{1} / 3+\hat{\zeta}_{2} / 2
$$

where $\hat{\zeta}, \hat{\zeta}_{0}, \hat{\zeta}_{1}$ and $\hat{\zeta}_{2}$ are given by Equations 8 through 11. For any $t$,

$$
P=\zeta+\sum_{i=2}^{t} p_{i} \text {. }
$$

$P$ can also be estimated, assuming Equation 12 holds, as is illustrated below. In many instances, this will yield the same estimate of $P$ as is given by Equation 14, but this is not always the case.

Table 1

Four Possible Outcomes When an Examinee Attempts an Item

\begin{tabular}{cccc}
\hline & \multicolumn{2}{c}{ Decision } & \\
\cline { 2 - 3 } $\begin{array}{r}\text { Latent } \\
\text { State }\end{array}$ & Knows & Doesn't Know & $\begin{array}{c}\text { Marginal } \\
\text { Probabilities }\end{array}$ \\
\cline { 2 - 3 } $\begin{array}{r}\text { Doesn't } \\
\text { Know }\end{array}$ & $\zeta_{0} / 4+\zeta_{1} / 3+\zeta_{2} / 2$ & $3 \zeta_{0} / 4+2 \zeta_{1} / 3+\zeta_{2} / 2$ & $\zeta$ \\
\hline
\end{tabular}


Using Equation 13, it would seem that for any fixed $\zeta$, the accuracy of an item is maximized when guessing is at random, i.e., when $\zeta_{1}=\zeta_{2}=0$ and $\zeta_{0}=1-\zeta$. This can be established in a more formal manner as follows: The inequality

$$
\Sigma b_{i} x_{i} \leq \Sigma c_{i} x_{i}
$$

holds whenever $x_{1} \leqslant x_{2} \leqslant \ldots \leqslant \mathrm{x}_{N}$ if and only if

$$
\sum_{i=1}^{k} c_{i} \geq \sum_{i=1}^{k} b_{i}, k=1, \ldots, n-1
$$

and $\Sigma c_{i}=\Sigma b_{i}$ (e.g., Marshall \& Olkin, 1979, p. 445). It follows that $P$ is maximized when $\zeta_{1}=\zeta_{2}=0$, since Equation 17 holds when $c=\left(\zeta, \zeta_{0}, \zeta_{1}, \zeta_{2}\right)$ and $b=(\zeta, 1-\zeta, 0,0)$.

Another way to characterize Table 1 is to use the "del" measure developed by Hildebrand, Laing, and Rosenthal (1977), which for the situation at hand is equivalent to Cohen's kappa (Cohen, 1960). In terms of the $\xi$ 's, this measure of association is

$$
\kappa=1-(1-P) / B
$$

where

$$
B=\left(\frac{{ }^{3 \zeta_{0}}}{4}+\frac{2 \zeta_{1}}{3}+\frac{\zeta_{2}}{2}\right)+\left(\zeta_{0}+\zeta_{1}+\zeta_{2}\right)\left(\zeta+\frac{\zeta_{0}}{4}+\frac{\zeta_{1}}{3}+\frac{\zeta_{2}}{2}\right) .
$$

Following Hildebrand et al. (1977), $x$ can be interpreted as follows: Suppose it is desired to measure the extent to which an examinee's latent state can be "predicted" according to the decision rule being used. The off-diagonal cells in Table 1 represent the error rates. The index $x$ represents the proportional reduction in the number of cases in the pair of error cells when a shift is made from statistical independence with the population marginals to the actual probability structure. Note that Equation 18 is the value of $x$ assuming the model holds.

\section{A Measure of Item "Idealness"}

Weitzman (1970) has described an asymptotic test of whether an item is ideal. As previously indicated, an item is defined to be ideal if guessing is at random. In the above notation, this corresponds to having $\zeta_{1}=\zeta_{2}=0$, which implies that $p_{2}=p_{3}=p_{4}$. A practical problem is that the null hypothesis that $p_{2}=p_{3}=p_{4}$ might be tested and rejected, when in fact $p_{2}, p_{3}$, and $p_{4}$ are nearly the same in value. This, in turn, might lead to efforts in improving the distractors when the item is already close to being ideal.

The simplest approach to this problem is to estimate $\zeta_{1}$ and $\zeta_{2}$ and to see how close they are to zero. If they are not, simply examine the distractors and decide whether any of them can be improved. Some additional possibilities are described and illustrated below.

When trying to determine whether $\xi_{1}$ and $\zeta_{2}$ are both close to zero, it might be desirable to take into account their combined effect on how close the item is to being ideal. Looking at $\zeta_{1}$ and $\zeta_{2}$ separately, they might appear to be close to zero; but together, perhaps, the item could be improved by a substantial amount. The problem becomes more complex when more than three distractors are used. Thus, it would be convenient to have some measure of how well an item approximates the ideal situation where $\xi_{0}=1-\zeta$.

One approach is to estimate $\zeta$, which yields an estimate of the proportion of agreement in Table 1 for the case $\zeta_{0}=1-\zeta$. Thus, the maximum possible value of $P$ for fixed $\zeta$ is estimated, say $P_{\max }$, which 
corresponds to the estimated value of $\zeta$. For $t=4, P_{\max }=3 / 4+\zeta / 4$. Next, estimate $P$, which yields an estimate of

$$
\Delta=P_{\max }-P
$$

This gives a measure of how ideal the item really is. When the model holds, $\Delta \geqslant 0$; and the closer $\Delta$ is to zero, the better the item.

Employing the $\Delta$ measure seems to be intuitively appealing, and in some situations it might suffice. However, there are at least two objections to its use. First, it has been suggested (e.g., Marshall \& Olkin, 1979, p. 408) that measures of inequality should have certain properties, namely, they should be Schur-convex, or strictly Schur-convex. ${ }^{1}$ Here the goal is to measure the inequality of $p_{2}, p_{3}$, and $p_{4}$. This requirement was first formulated by Dalton (1920), and steps in this direction were taken by Lorenz (1905) and Pigou (1912). Thus, as a measure of the inequality of $p_{2}, p_{3}$ and $p_{4}, \Delta$ might be objectionable because it is not Schur-convex. To see this, it is sufficient to observe that $\Delta$-as a function of $p_{2}, p_{3}$, and $p_{4}$-is not symmetric.

The second objection is that even when the model holds, the estimate of $\xi_{1}$ and $\xi_{2}$ can be negative, and the estimate of $\zeta_{0}$ can be greater than one. In this case, $\Delta$ cannot be interpreted as a difference of two probabilities. Perhaps $\Delta$ could be used anyway, but an investigator might prefer to use a more traditional index of inequality.

For the problem at hand, the index of inequality that suggests itself is the entropy function. The entropy of a probability mass function $p_{k} \geqslant 0, k=1, \ldots, r$, is

$$
H\left(p_{1}, \ldots, p_{r}\right)=-\Sigma p_{k} \log _{e} p_{k}
$$

where $\Sigma p_{k}=1$. (In some instances, the logarithms in Equation 21 are taken to the base 10 or the base 2 ; see Kullback, 1959 , p. 7.) The function $H$ provides a measure of the degree of uniformness of a distribution. That is, the larger $H$ is, the more uniform the distribution. The minimum value of $H$ occurs when $p_{1}=1$; its maximum value occurs when $p_{1}=\ldots p_{r}=1 / r$; and it is Schur-concave (implying that $-H$ is Schur-convex; see Marshall \& Olkin, 1979, chap. 13, sec. E). To measure the idealness of an item, the inequality of $p_{2}, p_{3}$, and $p_{4}$ needs to be measured, which suggests that $H\left(q_{1}, q_{2}, q_{3}\right)$ be used where $q_{i}=p_{i+1} /\left(1-p_{1}\right), i=1,2,3$. In this case, the maximum possible value of $H$ occurs when $q_{i}=1 /(t-1)$. An additional reason for using the entropy function is given in the next section of the paper. Brown (1965, sec. 3) also used the entropy function but in a slightly different fashion.

\section{Empirical Checks on the Model}

From Equations 1 through 4, various restrictions on the $p_{i}$ 's are evident in order for the model to hold. For instance, it requires having $p_{1} \geqslant p_{2} \geqslant p_{3} \geqslant p_{4}$. This assumption can be tested using results reported by Robertson (1978). It should be noted that when $p_{1}=p_{2}$, the probability of having $x_{1} \geqslant x_{2}$ approaches .5 as $N$, the number of examinees, gets large. Thus, there is a reasonably high probability that the usual estimate of the $p_{i}$ 's will indicate that the model does not hold when the $p_{i}$ 's are approximately equal in value. Of course, the hypothesis $p_{2}=p_{3}=p_{4}$ can be tested, but this does not give a direct measure of how ideal an item is. The null hypothesis might be rejected, for example, but this does not directly indicate the extent to which $p_{2}, p_{3}$, and $p_{4}$ are unequal. Another approach might be (1) to estimate $H$, especially when the data suggests the model might not hold and (2) if $H$ is reasonably close to its maximum value, to decide that the item is ideal. It is not suggested that hypothesis testing

\footnotetext{
'The meaning of a Schur-convex function is not given, since it does not play a direct role in the results to follow. The interested reader is referred to Marshall and Olkin (1979, chap. 3).
} 
be discarded altogether; the point is that the entropy function gives some additional information that is otherwise unavailable about how close an item is to being ideal. It might help to note that a similar situation occurs in the analysis of variance (Hays, 1973, pp. 484-488).

Another requirement of the model is that $p_{4} \leqslant 1 / 4$, otherwise, $\zeta_{0}>1$. For similar reasons the model requires that $p_{3}-p_{4} \leqslant 1 / 3$ and $p_{2}-p_{3} \leqslant 1 / 2$. However, $p_{1} \geqslant p_{2} \geqslant p_{3} \geqslant p_{4}$ implies that these additional inequalities are true.

\section{Mllustrations}

The results given above are illustrated with test scores for students enrolled in an undergraduate psychology course at the University of Southern California. Each item had $t=5$ distractors. There were four test forms, and each form had 40 items. For simplicity, only 4 items are analyzed and only one test form is used. A more extensive analysis of the data, together with some new theoretical results, will appear in a forthcoming report.

Table 2 gives the observed frequencies of the number of examinees who answered the item correctly on the $\mathrm{i}^{\mathrm{ith}}$ attempt $(i=1, \ldots, 5)$. For example, there were 42 examinees who were incorrect on their first attempt but were correct on their second attempt of Item 2 .

The first step when applying the results given above is to test the hypothesis that Equation 12 holds. As previously mentioned, this is accomplished with results in Robertson (1978). This was done for all 40 items on the test using a .01 level of significance. For Items 1 and 2 in Table 2, applying Robertson's test is not necessary, since the estimate of the $p_{i}$ 's already satisfies Equation 12. Item 3 was highly nonsignificant, but the null hypothesis was rejected for Item 4.

For 21 of the 40 items, Robertson's test was unnecessary, since the estimate of the $p_{i}$ 's satisfied Equation 12. For the remaining items, the null hypothesis was rejected only once; this was for Item 4 in Table 2.

Table 2

Number of Examinees Who are Correct on the ith Attempt of the Item

\begin{tabular}{crrrrr}
\hline & \multicolumn{5}{c}{ Attempt } \\
\cline { 2 - 6 } Item & 1 & 2 & 3 & 4 & 5 \\
\hline 1 & 139 & 14 & 9 & 4 & 2 \\
2 & 100 & 42 & 17 & 6 & 3 \\
3 & 68 & 34 & 16 & 29 & 21 \\
4 & 31 & 93 & 20 & 15 & 9 \\
\hline
\end{tabular}

Next, suppose a test constructor wants to determine whether a conventional scoring procedure will yield reasonably accurate decisions about whether an examinee has acquired the skills represented by Items 1,2 , and 3 in Table 2 . An estimate of $P$ via Equation 15 yields a partial solution to this problem. For Items 1 and 2 , the estimate of $\zeta$ is $(139-14) / 168=.744$ and $(100-42) / 168$ $=.345$, respectively. Thus, the corresponding estimates of $P$ are .917 and .75 .

As for Item 3, estimating $p_{3}$ and $p_{4}$, under the assumption that Equation 12 holds, requires an application of the pool-adjacent-violators algorithm in Barlow et al. (1972, pp. 13-18). The result is $\hat{p}_{3}=\hat{p}_{4}=(29+16) /(2(168))=.134$. The estimate of $\xi$ is .202 , and so the estimate of $P$ is .797. Note that using the pool-adjacent-violators algorithm yields the same estimate of $P$ as is obtained when 
Equation 15 is used and when $p_{i}$ is estimated with $x_{i} / n$. However, when $x_{1}<x_{2}$, using $\hat{p}_{i}=x_{i} / N$ will yield different results. The reason is that the maximum likelihood of $\zeta$, assuming Equation 12, is $\hat{\zeta}=0$ when $x_{1}<x_{2}$, and it is $\left(x_{1}-x_{2}\right) / N$ otherwise. Consider, for example, Item 4 in Table $2 . \hat{\zeta}=0$, and the maximum likelihood estimate of $p_{2}$, assuming Equation 12, is .369. Thus, the estimate of $P$ is .63. If, however, $p_{i}=x_{i} / N$ is used, the estimate of $P$ is .446 .

Suppose the first three items in Table 2 constituted the whole test. Another important point is that the estimates of $P$ yield an estimate of $\gamma$, the expected number of correct decisions for the $\boldsymbol{n}$ items on the test. The estimate is simply the sum of the estimated $P$ values. For the case at hand, $\gamma$ is estimated to be 2.46 . Thus, when a conventional scoring procedure is used to determine whether an examinee knows the correct response to an item, the expected number of correct decisions for the first three items in Table 2 is estimated to be 2.46 .

If any of the $P$ values is small, one possible way to improve the item is to improve the distractors. For example, efforts might be made to improve the least frequently chosen distractor.

To measure the effectiveness of the distractors, the entropy function is applied. For Item 1 in Table 2, $q_{1}=.483, q_{2}=.31, q_{3}=.138$, and $q_{4}=.069$. Substituting these values into Equation 21 yields $H=1.172$. The maximum possible value of $H$ occurs when $q_{i}=.25(i=1,2,3,4)$, in which case $H=1.386$. For Item $2, H=.99$; and for Item $3, H=1.347$. Thus, the test scores indicate that the item with the most effective distractors is Item 3 , followed by Item 1 . The distractors for Item 2 are the least effective, having achieved $71.4 \%$ of the maximum possible entropy.

It should be pointed out that the above estimate of $H$ for Item 3 was not made under the assumption that Equation 12 holds. If Equation 12 is assumed, and the pool-adjacent-violators algorithm is applied, this yields $\hat{p}_{i}=.405, \hat{p}_{2}=\hat{p}_{3}=\hat{p}_{4}=.1568$, and $\hat{p}_{5}=.125$, in which case $H=1.382$. In either case, Item 3 has the most effective distractors.

\section{A Model for Type II Guessing}

In many instances a test consists of items representing skills that are thought to be most important. Moreover, there are situations in which the skills on a test are the only ones that are of interest to the test constructor. However, in other situations (see, e.g., Hambleton, Swaminathan, Algina, \& Coulson, 1978) the items on a test are intended to be a representative sample of some larger item domain. The goal is to use test results to make inferences about what an examinee knows relative to the item pool. In either case, the results in the previous section are of interest. This section considers how an AUC test might be used to solve certain measurement problems when generalizing results for a single examinee to an item domain.

For a specific examinee, let $\xi$ be the proportion of skills among a domain of skills that he/she has acquired. Further, suppose that each skill is represented by a multiple-choice test item having $t$ alternatives from which to choose. Again, for convenience, emphasis is given to the special case $t=4$. Let $\xi_{i}(i=0, \ldots, t-2)$ be the proportion of items for which the examinee does not know and can eliminate $i$ distractors. Once $i$ distractors are eliminated, the examinee is assumed to guess at random from among those that remain. Let $r_{i}$ be the probability of a correct response on the $i^{\text {th }}$ attempt. Then, for $t=4$,

$$
\begin{aligned}
& r_{1}=\xi_{0}+\xi_{0} / 4+\xi_{1} / 3+\xi_{2} / 2 \\
& r_{2}=\xi_{0} / 4+\xi_{1} / 3+\xi_{2} / 2 \\
& r_{3}=\xi_{0} / 4+\xi_{1} / 3
\end{aligned}
$$




$$
r_{4}=\xi_{0} / 4
$$

If for a random sample of $n$ items, $y_{i}$ is the number of items the examinee has correct on the $i^{\text {th }}$ alternative chosen, an unbiased estimate of the $\xi_{i}$ 's can be derived just as unbiased estimates of $\xi_{i}$ 's were derived in the previous model. In particular, an unbiased estimate of $\xi$ is

$$
\hat{\xi}=\left(y_{1}-y_{2}\right) / n
$$

Equation 26 is an estimate of true score that is corrected for an examinee's partial information. Note that Equation 26 contains the usual correction for guessing formula score as a special case.

\section{The Optimal Linear Estimator of $\xi$}

Let $z$ be a random variable that is an unbiased estimate of the unknown parameter $\theta$. Under squared error loss, Griffin and Krutchkoff (1971) show that the optimal linear estimator of $\theta$ is

$$
\hat{\theta}=\alpha z+\delta
$$

where $\alpha=\operatorname{Var}(\theta) / \operatorname{Var}(\mathrm{z})$ and $\delta=(1-\alpha) \mathrm{E}(\theta)$. In mental test theory, Equation 27 is known as Kelley's linear regression estimate of true score (Kelley, 1947, p. 409). The point made by Griffin and Krutchkoff (1971) is that if an unbiased estimate of an examinee's true score is used, Equation 27 is optimal regardless of the shape of true score distribution. Wilcox (1978) compares Equation 27 to several other estimators, assuming the binomial error model holds but where observed scores are generated according to a two-term approximation to the compound binomial error model. The results suggest that when simultaneously estimating the true score of several examinees, the Griffin-Krutchkoff (1971) estimator should be used when an ensemble squared error loss function is being used. Furthermore, the results suggest that Kelley's linear regression estimate of $\xi$ be employed.

It is assumed that the $y_{i}$ 's have a multinomial distribution and that observed test scores for $N$ examinees are available. An estimate of $\mathrm{E}(\xi), \operatorname{Var}(\xi)$, and $\operatorname{Var}\left(y_{1}-y_{2}\right)$ is needed to apply results in Griffin and Krutchkoff (1971), where the expectations defining these quantities are over the population of examinees.

$$
\begin{aligned}
& \text { Let } \\
& v_{i}=y_{i} / n \\
& w_{i}=\frac{y_{i}}{n} \quad \frac{y_{i}-1}{n-1}
\end{aligned}
$$

where $i=1,2$. Then

$$
\begin{aligned}
& E\left(v_{i} \mid p_{1}, p_{2}\right)=p_{i} \\
& E\left(w_{j} \mid p_{1}, p_{2}\right)=p_{i}^{2}
\end{aligned}
$$

Since $\operatorname{cov}\left(y_{1}, y_{2} \mid p_{1}, p_{2}\right)=-2 n p_{1} p_{2}$, it follows that

$$
E\left(y_{1} y_{2} \mid p_{1}, p_{2}\right)=n(n-2) p_{1} p_{2}
$$

Thus,

$$
E\left(v_{1}-v_{2}\right)=E(\xi)
$$


and

$$
E\left(w_{1}+w_{2}-\left[2 y_{1} y_{2} /(n(n-2))\right]\right)=E\left(\xi^{2}\right)
$$

Letting $y_{i j}$ and $v_{i j}$ be the true value of $y_{i}$ and $v_{i}$, respectively, for the $j^{\text {th }}$ randomly sampled examinee, the above results suggest that $E(\xi)$ be estimated with

$$
\hat{\mu}_{\xi}=N^{-1} \sum_{j=1}^{N}\left(v_{1 j}-v_{2 j}\right)
$$

and $E\left(\xi^{2}\right)$ with

$$
\hat{\tau}_{\xi}=N^{-1} \sum_{j=1}^{N} w_{1 j}+w_{2 j}-n^{-1}(n-2)^{-1} 2 y_{1 j} y_{2 j}
$$

Ihus, an estimate of $\overline{\operatorname{Var}(\xi)}$ is

$$
\hat{\sigma}_{\xi}^{2}=\hat{\tau}_{\xi}-\left(\hat{\mu}_{\xi}\right)^{2}
$$

The variance of the marginal distribution of observed scores $\left(y_{1}-y_{2}\right) / n$ can be estimated in the usual manner, and so an estimate of the optimal linear estimator of $\xi$ is obtained by substituting the results in Equation 27. Of course, the results just given contain, as a special case, the optimal linear estimator under the assumption guessing is at random.

\section{Numerical Illustration}

As a simple illustration, suppose there are five examinees with observed $y$ values as shown in the first two rows of Table 3 , where the test length is $n=10$. Then $\hat{\mu}_{t}=.42, \hat{\tau}_{\xi},=.2$, and so $\hat{\sigma}_{\xi}=.0236$. The estimate of $\operatorname{Var}\left(\left(y_{1}-y_{2}\right) / n\right)$ is .0687. Therefore, the estimate of $\alpha$ is $\hat{\alpha}=.3435$, and so the estimate of the optimal linear estimator is

$$
\tilde{\xi}=.3435\left(\mathrm{y}_{1}-\mathrm{y}_{2}\right) / \mathrm{n}+.276
$$

The values of $\tilde{\xi}$ for the five examinees are given in the last row of Table 3 .

\begin{tabular}{lccccc}
\multicolumn{7}{c}{ Table 3} \\
Values of $y_{1}, y_{2}$ & and $\stackrel{n}{\xi}$ With & $n=10$ \\
& and $N=5$ \\
$y_{1}$ & 5 & 7 & 5 & 9 & 2 \\
$y_{2}$ & 3 & 1 & 2 & 0 & 2 \\
$\tilde{\xi}$ & .34 & .48 & .47 & .59 & .28 \\
\hline
\end{tabular}

Before continuing, some additional comments about the above results are in order. First, the estimate of $\operatorname{Var}(\xi)$ can be negative, in which case $\alpha=1$ is used. The same phenomenon occurs in the case considered by Griffir and Krutchkoff (1971). Second, the optimal linear estimator of $\xi$ derived above 
does not assume the model holds. It is the optimal linear estimator of $p_{1}-p_{2}$, but no insistance is made that $p_{1} \geqslant p_{2}$. If the model holds, implying that $p_{1} \geqslant p_{2}$, Equation 33 is no longer true; and so the condition of having an unbiased estimate of $\xi$, as is assumed by Griffin and Krutchkoff, is no longer satisfied. (For further comments on this approach to estimation, see Griffin and Krutchkoff, 1971, and Wilcox, 1978.)

\section{A Strong True Score Model}

This section assumes that for any examinee, $y_{1}$ and $y_{2}$ have a multinomial probability function given by

$$
f\left(y_{1}, y_{2} \mid \xi, p_{2}\right)=\frac{n !\left(\xi+p_{2}\right)^{y_{1}} p_{p^{2}}^{y_{2}}\left(1-\xi-p_{2}\right)^{n-y_{1}-y_{2}}}{y_{1} ! y_{2} !\left(n-y_{1}-y_{2}\right) !}
$$

where, as before, $\xi=p_{1}-p_{2}$ and $0 \leqslant \xi=1$ is assumed. Equation 39 can be justified under an itemsampling model, or it might give a good approximation to the joint probability function of $y_{1}$ and $y_{2}$. It should be noted that Equation 39 implies that $y_{1}$ has a binomial probability function, and so when every examinee takes the same $n$ items, the items have the same level of difficulty (Lord \& Novick, 1968, chap. 23). On theoretical grounds, this implication of Equation 34 is unjustifiable. However, for certain measurement problems, it appears that this might not be a serious restriction (Algina \& Noe, 1978; Wilcox, 1977, 1978; see also Subkoviak, 1978).

Strong true-score models attempt to extend assumptions such as Equation 39 to a population of examinees. The basic problem here is to find a family of distributions that approximates $g\left(\xi, p_{2}\right)$, the joint density of $\xi$ and $p_{2}$. Once this is done, various measurement problems can be solved (e.g., Huynh, 1976; Lord, 1965; Wilcox, 1977).

Past experience with this type of problem (Keats \& Lord, 1962; Lord, 1965; Wilcox, 1979) suggests approximating $g\left(\xi, p_{2}\right)$ with a bivariate Dirichlet function given by

$$
\frac{\Gamma\left(v_{1}+v_{2}+v_{3}\right)}{\Gamma\left(v_{1}\right) \Gamma\left(v_{2}\right) \Gamma\left(v_{3}\right)} \quad \xi^{v_{1}} p_{2}^{v_{2}}\left(1-\xi-p_{2}\right)^{v_{3}}
$$

where $\Gamma$ is the usual gamma function, $v_{i}>0(i=1,2,3)$ are unknown parameters and $0 \leqslant \xi+p \leqslant 1$. (Marshall \& Olkin, 1979, pp. 306-307 describe two other distributions to which the name "Dirichlet" is attached. Here, only Equation 40 is considered.)

To estimate the $v_{i}$, proceed as follows: First, observe that the marginal distribution of $\xi$ is beta with parameters $v_{1}$ and $v_{2}+v_{3}$ (e.g., Wilks, 1962). It follows that

$$
v_{1}\left(1-\mu_{\xi}^{-1}\right)+v_{2}+v_{3}=0
$$

where, as before, $\mu_{\xi}$ is the mean of $\xi$ over the population of examinees. For similar reasons,

$$
v_{1}+v_{2}\left(1-\mu_{p}^{-1}\right)+v_{3}=0
$$

where $\mu_{p}$ is the mean of $p_{2}$. It is also known (e.g., Wilcox, 1977) that

$$
s=v_{2}+v_{3}
$$

where

$$
s=\mu_{\xi}\left(1-\mu_{\xi}\right)^{2} \sigma_{\xi}^{-2}+\mu_{\xi}-1 \text {. }
$$


Summarizing these results in matrix notation yields

$$
\left[\begin{array}{lll}
1-\mu_{s}-1 & 1 & 1 \\
1 & 1-\mu_{p}-1 & 1 \\
0 & 1 & 1
\end{array}\right]\left[\begin{array}{c}
v_{1} \\
v_{2} \\
v_{3}
\end{array}\right]=\left[\begin{array}{l}
0 \\
0 \\
s
\end{array}\right]
$$

As previously indicated, $\mu_{\xi}$ and $\mu_{\xi}^{2}$ can be estimated, which yields an estimate of $s$. An estimate of $p_{2}$ is $N^{-1} \Sigma y_{2 j}$, and so Equation 44 yields an estimate of the $v_{i}$ 's.

Mosimann (1962) applies the Dirichlet-multinomial model to two real data sets, discusses how to check the implications of the model, and gives several other results that have practical value; thus, these issues are not discussed further. Since the Dirichlet-multinomial model is the multivariate analog of the beta-binomial model, additional insights into the appropriateness of the model are available from Wilcox (in press-a). The point is that the Dirichlet-multinomial model can be applied to AUC scoring procedures and so can solve various measurement problems, as previously indicated. An advantage of the model is that it allows guessing to vary over the population of examinees.

An important point is that if the model is assumed to hold, and in particular $0 \leqslant \xi \leqslant 1$, this suggests estimating $\xi$ to be zero even when $\hat{\xi}<0$. In this case, the estimates of $E(\xi)$ and $E\left(\xi^{2}\right)$ are not justified for the reasons given above, but they are still appropriate for the reasons given by Wilcox (1979).

One point that deserves special mention is that a new formula score can be derived that corrects for partial information. The derivation is essentially the same as the derivation of Equation 4 in Wil$\operatorname{cox}$ (1979). Thus, it is merely noted that

$$
E\left(\xi \mid y_{1}\right)=\left[f\left(y_{1}\right) B\left(v_{1}, v_{2}, v_{3}\right)\right]^{-1} \quad\left(\begin{array}{l}
n \\
y_{1}
\end{array}\right)
$$

$$
\sum_{w=0}^{y_{1}} B\left(w+v_{2}, n-y_{1}+v_{3}\right) B\left(y_{1}-w+v_{1}+1, n-y_{1}+w+v_{2}+v_{3}\right)
$$

where $B$ is the usual beta function. Thus, once the $v_{i}$ 's are estimated, only $y_{1}$ is needed to estimate $\xi$.

\section{Discussion}

One objection to the assumptions that were made is that the resulting model is too simple. For instance, it does not allow for the possibility of knowing and being incorrect or the possibility of having misinformation. Brown and Burton (1979) describe a real situation in which the latter problem occurs. Frary (1980) gives an interesting account of how misinformation can affect various scoring procedures, and Wilcox (1980) indicates the seriousness of the former problem when determining the length of a criterion-referenced test. Although the present model does not correct these problems, empirical checks on the appropriateness of the model can be made. It should be mentioned that models have been proposed for handling the two errors just described (e.g., Dayton \& Macready, 1976; Duncan, 1974; Macready \& Dayton, 1977). However, these models require additional assumptions that might not be met. The Macready-Dayton (1977) model, for example, assumes that equivalent items are available for measuring a particular skill. The assumption of equivalent items can be checked us- 
ing a goodness-of-fit test (Macready \& Dayton, 1977), using a procedure described by Hartke (1978); and results reported by Baker and Hubert (1977) might also be useful in this endeavor (see also Wilcox, in press-b). Here it is assumed that empirical investigations fail to support the existence of equivalent items, or it is decided a priori that equivalent items do not exist. Finally, the Duncan (1974) model corrects for misinformation, but it assumes guessing is at random. The goal here is to avoid this restriction or to find ways in which it can be empirically checked.

Another possible objection to the model is that it characterizes examinees as belonging to one of two mutually exclusive classes, namely, "knowing" and "not knowing." The relative merits of this approach are discussed in a more general context by Reulecke (1977), Hilke, Kempf, and Scandura (1977), Scandura (1971, 1973), and Spada (1977).

In some situations, the scoring procedure for Type II guessing might be objectionable because it penalizes an examinee for having partial information. That is, if an examinee wants to maximize his/her score (the estimate of $\xi$ ) the strategy would be to minimize $y_{2}$. This could be done by choosing an answer, and if it is wrong, by deliberately choosing another response that is believed to be incorrect. In this case, the examinee is not behaving in the manner assumed, and so the model is inappropriate. One approach to this problem is to have an examinee always mark his/her first and second choice without revealing which response is correct. Letting $y_{1}$ be the number of times the examinee's first choice is correct, and letting $y_{2}$ be the number of times the second choice is correct, $\xi$ is again estimated with $\left(y_{1}-y_{2}\right) / n$. Indeed, all of the previous results still hold.

However, this might not eliminate the problem under discussion. Suppose, for example, that an examinee can eliminate all but two of the alternatives from consideration for every item on the test. If an examinee's two choices correspond to these two alternatives, the expected estimate of $\xi$ is 0 . However, if an examinee's first choice is between the two alternatives that contains the correct response, and if the examinee is deliberately incorrect on the second choice, the expected estimate of $\xi$ is .5. One way to minimize this problem is to subject the items to an analysis that attempts to ensure that guessing is at random. It was already indicated how this might be done. Another solution is to apply the Dirichlet-multinomial model. If estimates of the $v_{i}$ 's can be made available, the information on the examinee's first choice (the value of $y_{1}$ ) is all that is needed in order to estimate $\xi$. Several other strong true-score models are currently being investigated that might be useful when addressing this problem. Another possibility is to check the assumptions of the model; if they do not hold, simply score the test using traditional techniques.

For practical purposes, perhaps the problem just described will be inconsequential; this remains to be seen. Also note that this problem is irrelevant in terms of the results given under Type I guessing.

In practice, the scoring rule proposed by Brown (1965) results in scoring $t-i$ points when the correct response is chosen on the $i^{\text {th }}$ attempt of an item, where, as before, $t$ is the number of alternatives from which to choose (e.g., Frary, 1980). Thus, the sooner an examinee identifies the correct answer, the higher his/her score will be. In some cases, however, this scoring procedure is also inadequate. First, it gives credit to an examinee when a test constructor unintentionally produces ineffective distractors. Second, and perhaps most importantly, it gives a measure of partial information, but it does not tell us what an examinee knows in the sense of estimating $\xi$. The same is true of the other scoring procedures cited by Frary (1980), the scoring rule proposed by Coombs, Milholland, and Womer (1956), as well as the subset selection rule proposed by Gibbons, Olkin, and Sobel (1977, 1979). No claim is made that these procedures be abandoned; but, as argued by Morrison and Brockway (1979), estimating $\xi$ can be important.

Another point is that only two responses to each item are needed in order to estimate $\xi$ for each examinee. The additional responses are needed only for checking the appropriateness of the model, 
and in particular for justifying $\left(y_{1}-y_{2}\right) / n$ as an estimate of $\xi$. In some cases, $n$ will be too small to test the model accurately. Determining whether this is the case can be accomplished with the statistical techniques described under Type I guessing.

Finally, it was suggested that the Dirichlet-multinomial distribution be considered when trying to find a strong true-score model that fits the data. It should be stressed, however, that considerably more experience with this distribution is needed before it is routinely applied. Wilcox (in press-c) obtained good results with the distribution using real data, but the extent to which it gives a good fit to mental test data is not known. An empirical investigation is currently underway in an attempt to partially resolve this problem. Consideration will also be given to several other strong true-score models.

\section{References}

Algina, J., \& Noe, M. G. A study of the accuracy of Subkoviak's single-atiministration estimate of the coefficient of agreement using two true-score estimates. Journal of Educational Measurement, $1978,15,101-110$.

Baker, F. B., \& Hubert, L. J. Inference procedures for ordering theory. Journal of Educational Statistics. 1977, 2, 217-233.

Barlow, R. E., Bartholomew, D. J., Bremner, J. M., \& Brunk, H. D. Statistical inference under order restrictions. New York: Wiley, 1972.

Bliss, L. B. A test of Lord's assumption regarding examinee guessing behavior on multiple-choice tests using elementary school students. Journal of Educational Measurement, 1980, 17, 147-153.

Brown, J. Multiple response evaluation of discrimination. The British Journal of Mathematical and Statistical Psychology, 1965, 18, 125-137.

Brown, J. C., \& Burton, R. R. Diagnostic models in basic mathematical skills, In National Institute of Education, Testing, Teaching, and Learning, Report of a Conference on Research on Testing. Washington DC: U.S. Department of Health, Education and Welfare, 1979.

Cohen, J. A coefficient of agreement for nominal scales. Educational and Psychological Measurement, 1960, 20, 37-46.

Coombs, C. H., Milholland, J. E., \& Womer, F. B. The assessment of partial knowledge. Educational and Psychological Measurement, 1956, 16, 13-37.

Cross, L. H., \& Frary, R. B. An empirical test of Lord's theoretical results regarding formula scoring of multiple-choice tests. Journal of Educational Measurement, 1977, 14, 313-321.

Dalton, $\mathrm{H}$. The measurement of the inequality of incomes. Economic Journal, 1920, 30, 348-361.

Dayton, C. M., \& Macready, G. B. A probabilistic model for validation of behavioral hierarchies. Psychometrika, 1976, 41, 189-204.

Duncan, G. T. An empirical Bayes approach to scoring multiple-choice tests in the misinformation model. Journal of the American Statistical Association, 1974, 69, 50-57.

Frary, R. B. The effect of misinformation, partial information, and guessing on expected multiplechoice test item scores. Applied Psychological Measurement, 1980, 4, 79-90.

Gibbons, J., Olkin, I., \& Sobel, M. Selecting and ordering populations: A new statistical methodology. New York: Wiley, 1977.

Gibbons, J. D., Olkin, I., \& Sobel, M. A subset selection technique for scoring items on a multiple choice test. Psychometrika, 1979, 44, 259-270.

Gilman, D. A., \& Ferry, P. Increasing test reliability through self-scoring procedures. Journal of Educational Measurement, 1972, 9, 205-207.

Griffin, B. S., \& Krutchkoff, R. G. Optimal linear estimators: An empirical Bayes version with application to the binomial distribution. Biometrika. 1971, 58, 195-201.

Hambleton, R. K., Swaminathan, H., Algina, J., \& Coulson, D. Criterion-referenced testing and measurement: A review of technical issues and developments. Review of Educational Research. 1978, 48, 1-47.

Hanna, G. S. Incremental reliability and validity of multiple-choice tests with an answer-until-correct procedure. Journal of Educational Measurement, 1975, 12, 175-178.

Harris, C. W., \& Pearlman, A. P. An index for a domain of completion or short answer items. Journal of Educational Statistics, 1978, 3, 285-304.

Hartke, A. R. The use of latent partition analysis to identify homogeneity of an item population. Journal of Educational Measurement. 1978, 15, 43-47.

Hays, W. Statistics for the social sciences. New York: Holt, Rinehart \& Winston, 1973.

Hildebrand, D. K., Laing, J. D., \& Rosenthal, H. Prediction analysis of cross classifications. New York: Wiley, 1977.

Hilke, R., Kempf, W. F., \& Scandura, J. M. Deterministic and probabilistic theorizing in structural 
learning. In H. Spada \& F. Kempf (Eds.), Structural models of thinking and learning. Bern: Huber, 1977.

Horst, P. The difficulty of a multiple choice test item. Journal of Educational Psychology, 1933, 24, 229-232.

Huynh, H. Statistical consideration of mastery scores. Psychometrika, 1976, 41, 65-78.

Keats, J. A., \& Lord, F. M. A theoretical distribution for mental test scores. Psychometrika, 1962, 27 , 59-72.

Kelley, T. L. Fundamentals of statistics. Cambridge: Harvard University Press, 1947.

Kullback, S. Information theory and statistics. New York: Wiley, 1959.

Lord, F. M. A strong true-score theory, with applications. Psychometrika, 1965, 30, 239-270.

Lord, F. M., \& Novick, M. R. Statistical theories of mental test scores. Reading MA: Addison-Wesley, 1974.

Lorenz, M. O. Methods of measuring concentration of wealth. Journal of the American Statistical Association, 1905, 9, 209-219.

Macready, G. B., \& Dayton, C. M. The use of probabilistic models in the assessment of mastery. Journal of Educational Statistics, 1977, 2, 99-120.

Marshall, A. W., \& Olkin, I. Inequalities: Theory of majorization and its applications. New York: Academic Press, 1979.

Morrison, D. G., \& Brockway, G. A modified beta-binomial model with applications to multiple choice and taste tests. Psychometrika, 1979, 44, 427-442.

Mosimann, J. E. On the compound multinomial distribution, the multivariate $\beta$-distribution, and correlations among proportions. Biometrika, 1962, 49, 65-82.

Pigou, A. C. Wealth and welfare. New York: Macmillan, 1912.

Pressey, S. L. Development and appraisal of devices providing immediate automatic scoring of objective tests and concomitant self-instruction. The Journal of Psychology, 1950, 29, 419-447.

Reulecke, W. A. A statistical analysis of deterministic theories. In H. Spada \& F. Kempf (Eds.), Structural models of thinking and learning. Bern: Huber, 1977.

Robertson, T. Testing for and against an order restriction on multinomial parameters. Journal of the American Statistical Association, 1978, 73, 197-202.

Scandura, J. M. Deterministic theorizing in structural learning. Journal of Structural Learning, 1971, 3, 21-53.

Scandura, J. M. Structural learning: Theory and research. New York: Gordon \& Breach, 1973.
Spada, H. Logistic models of learning and thought. In H. Spada \& F. Kempf (Eds.), Structural models of thinking and learning. Bern: Huber, 1977.

Subkoviak, M. Empirical investigation of procedures for estimating reliability for mastery tests. Journal of Educational Measurement, 1978, 15. 111-116.

Weitzman, R. A. Ideal multiple-choice items. Journal of the American Statistical Association, 1970, 65, 71-89.

Wilcox, R. R. New methods for studying stability. In C. W. Harris, A. Pearlman, \& R. Wilcox, Achievement test items-Methods of study (CSE Monograph No. 6). Los Angeles: University of California, Center for the Study of Evaluation, 1977. (a)

Wilcox, R. R. Estimating the likelihood of false-positive and false-negative decisions in mastery testing: An empirical Bayes approach. Journal of Educational Statistics, 1977, 2, 289-307. (b)

Wilcox, R. R. Estimating true score in the compound binomial error model. Psychometrika, 1978, 43, 245-258.

Wilcox, R. R. Achievement tests and latent structure models. British Journal of Mathematical and Statistical Psychology, 1979, 32, 61-71.

Wilcox, R. R. Determining the length of a criterionreferenced test. Applied Psychological Measurement, 1980, 4, 425-446.

Wilcox, R. R. A review of the beta-binomial model and its extensions. Journal of Educational Statistics, in press. (a)

Wilcox, R. R. Analyzing the distractors of multiplechoice test items or partitioning multinomial cell probabilities with respect to a standard. Educational and Psychological Measurement, in press. (b)

Wilcox, R. R. The single administration estimate of the proportion of agreement of a proficiency test scored with a latent structure model. Educational and Psychological Measurement, in press. (c)

Wilks, S. S. Mathematical statistics. New York: Wiley, 1962.

Zehna, P. W. Invariance of maximum likelihood estimation. Annals of Mathematical Statistics, 1966, 37, 744.

\section{Acknowledgments}

The author thanks Scott Fraser for generously supplying the data used in this study and Joan Murray for helpful comments on an earlier draft of this paper. The project reported herein was performed pursuant to a grant fron the National Institute of Education, Department of Health, Education, and Welfare. 
However, the opinions expressed do not necessarily reflect the position or policy of the National Institute of Education, and no official endorsement by the $\mathrm{Na}$ tional Institute of Education should be inferred.

\section{Author's Address}

Send requests for reprints or further information to Rand R. Wilcox, Department of Psychology, University of Southern California, Los Angeles CA 90007. 